\title{
Ex vivo lung perfusion: The magic bullet to cure sick donor lungs?
}

\author{
Florian M. Wagner, MD, and Hermann Reichenspurner, MD, $\mathrm{PhD}$
}

\author{
From the Department of Cardiovascular Surgery, University Heart Center Hamburg, Hamburg, Germany. \\ Disclosures: Authors have nothing to disclose with regard to commercial support. \\ Received for publication Oct 14, 2015; accepted for publication Oct 14, 2015. \\ Address for reprints: Hermann Reichenspurner, $\mathrm{MD}, \mathrm{PhD}$, Department of Cardiovascular Surgery, University \\ Hospital Eppendorf, Martinistrasse 52, D-20246 Hamburg, Germany (E-mail: hor@uke.uni-hamburg.de). \\ J Thorac Cardiovasc Surg 2016;151:547-8 \\ $0022-5223 / \$ 36.00$ \\ Copyright (c) 2016 by The American Association for Thoracic Surgery \\ http://dx.doi.org/10.1016/j.jtcvs.2015.10.052
}

In this issue of the Journal, Wagner and colleagues from the University of Virginia report about the effects of an adenosine $\mathrm{A} 2 \mathrm{~A}$ receptor agonist administered to donor after cardiac death lungs exposed to 12 hours of cold ischemia and reconditioned for 4 hours in an ex vivo lung perfusion (EVLP) circuit before being transplanted in a unilateral porcine model. Wagner and colleagues hypothesized that this drug reduces the inflammatory response within an allograft, mitigates ischemia-reperfusion injury, and improves outcome after lung transplant even under these challenging circumstances.

In the first place, Wagner and colleagues must be congratulated for their tremendous efforts to translate a complex clinical situation into a sophisticated large animal model. This model still has significant limitations, however, which need to be considered before interpreting the observed data. Unilateral lung transplant without excluding support of the remaining native (healthy) lung renders interpretation of allograft function complicated, if not close to impossible. As acknowledged by Wagner and colleagues none of the transplanted donor lungs achieved functional values during EVLP that are currently considered necessary to allow transplant in a clinical setting. ${ }^{1}$ After exposure to a second ischemic period, function will at best remain similar if not further deteriorate during reperfusion in the recipient. The majority of blood flow and ventilation therefore will be directed into the recipient's remaining native lung. To date it has been recognized that gas exchange alone poorly reflects the degree of primary graft dysfunction ${ }^{2}$; however, it becomes absolutely unreliable in this animal model with unclear participation of allograft perfusion and ventilation. Reported mean wet/dry ratios between 8 and 13 (normal range, 4-5) clearly indicate significant edema; this is accompanied by increased cellular infiltrations, as can be seen in the included histologic samples. This is in contrast to the assumption by Wagner and colleagues of improved grade of primary graft dysfunction in A2A-treated lungs! Furthermore, because of the lack of a true control group (lung transplant after 12 hours of cold ischemia without EVLP and A2A

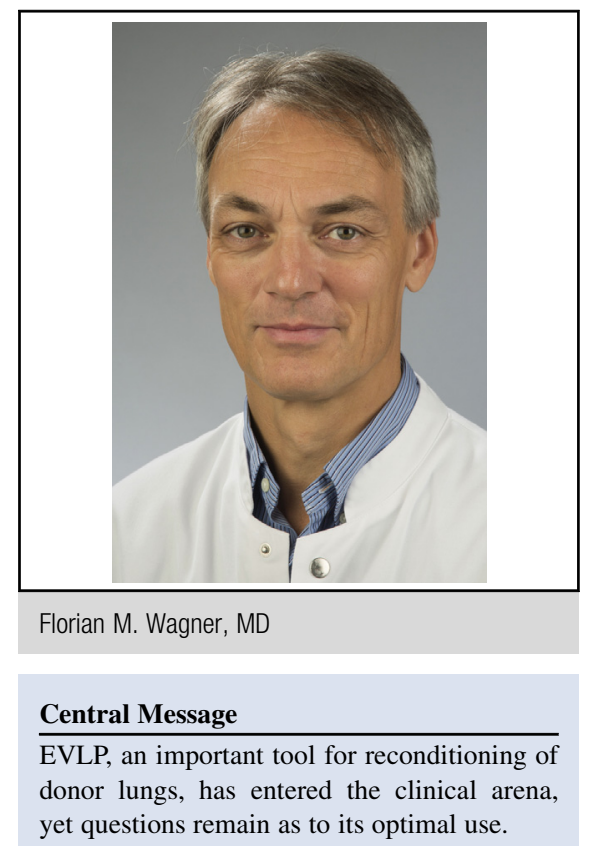

See Article page 538.

See Editorial page 315 .

agonist), interpretation of the potential beneficial effects of taken measures remain highly speculative.

In this study, EVLP was mainly used and considered as an "ideal platform for isolated drug delivery" to the graft. This is certainly an important facet of EVLP, but most research has so far focused on treatment strategies to correct pathophysiologic changes in donor lungs that are sequelae of brain death or exposure to an agonal phase after cardiac arrest. Almost 2 decades ago, Steen and coworkers ${ }^{3}$ started their research to develop a platform for ex vivo reconditioning of donor after cardiac death lungs to report the first successful clinical transplant in 2001. Since then, EVLP has become a recognized concept to repair not only donor after cardiac death lungs but also marginal lungs from brain-dead donors, allowing significant increase in quantity of suitable lungs for transplant without increased incidence of primary graft dysfunction. ${ }^{4}$ Yet many open questions remain. This ranges from the discussion of which are the most important and suitable functional parameters during EVLP to judge transplantability to questions about ideal perfusate composition, perfusion protocols, open versus closed system, and finally to the point what is adequate or maximum duration of safe perfusion. It is therefore not surprising that reported rates of lungs considered suitable 
for transplantation after EVLP vary significantly, from $45 \%$ to $100 \%$. So far we have learned, thanks to systematic research by the Toronto group and others, that we can extract edema, remove atelectasis, and normalize ventilation/perfusion patterns by sole perfusion with a highly oncotic acellular crystalloid solution. Experimentally, Cyphel and colleagues 5 found this technique to be safe for perfusion times as long as 12 hours. In a clinical scenario, however, most investigators keep these times as short as possible, with most protocols ending after 4 hours of EVLP, despite experimental data indicating potential benefit beyond this time frame. ${ }^{6}$ It remains speculative whether more complex pathologic conditions of donor lungs can also be corrected, and if so whether this requires extended protocols including more physiologically composed perfusates similar to blood as well as systematic removal of toxic metabolites during EVLP.

For the time being, research should rather focus on a better understanding of basic mechanisms, such as relevance of easily available functional parameters and perfusion times, before we start pushing limits within complex animal models. Once we have learned the best ways to perfuse, ventilate, and manage donor lungs within an EVLP setting, we should start applying sophisticated drug and gene therapy. But let's first learn to walk before we start running. After all, EVLP technology is currently the most promising tool and of tremendous importance to expand lung transplantation, particularly in the setting of increasingly complex and older donor situations.

\section{References}

1. Cyphel M, Yeung JC, Liu M, Anraku M, Chen F, Karolak W, et al. Normothermic ex vivo lung perfusion in clinical lung transplantation. N Engl J Med. 2011;364: 1431-40.

2. Yeung JC, Cyphel M, Machuca TN, Koike T, Cook DJ, Bonato R, et al. Physiologic assessment of the ex vivo donor lung for transplantation. J Heart Lung Transplant. 2012;31:1120-6.

3. Steen S, Sjöberg T, Pierre L, Liao Q, Erikssson L, Algotsson L. Transplantation of lungs from a non-heart-beating donor. Lancet. 2001;351:825-9.

4. Andreasson AS, Dark JH, Fisher AJ. Ex vivo lung perfusion in clinical lung transplantation-state of the art. Eur J Cardiothorac Surg. 2014;46:779-88.

5. Cyphel M, Yeung JC, Hirayama S, Rubacha M, Fischer S, Anraku M, et al. Technique for prolonged normothermic ex vivo lung perfusion. J Heart Lung Transplant. 2008;27:1319-25.

6. Wipper S, Martens B, Dupree A, Schirmer J, Pahrmann C, Reichenspurner H, et al. Development and evaluation of an in-vitro reperfusion circuit for reconditioning of predamaged donor-lungs. J Heart Lung Transplant. 2008;27:S201. 